\title{
Innovative phytotherapeutische Ansätze - auf Entdeckungsreise in Zürich
}

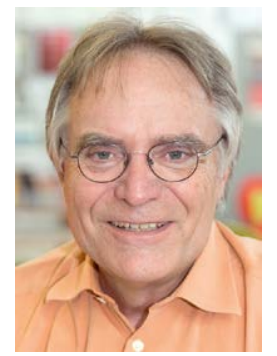

Prof. Dr. Beat Meier
Einladung zur tetranationalen Tagung „Phytotherapie 2022 - innovativ“ vom 16.-18. Juni in Zürich

Oft ist die Rede davon, dass in der Phytotherapie keine Innovationen mehr möglich sind. Ja, es trifft zu, dass kaum mehr Arzneimittel aus der Phytotherapie mit neuen Anwendungsgebieten zugelassen werden. Zu groß ist der Aufwand dafür geworden. Stattdessen sind den Nahrungsergänzungsmitteln und Medizinprodukten fast keine kreativen Grenzen gesetzt. Das heißt aber nicht, dass die Phytotherapie nicht mit innovativen Ansätzen aufwarten kann.

Die tri-, seit 2018 tetranationale Tagung der Phytotherapie-Gesellschaften von Deutschland, Niederlande, Österreich und der Schweiz hat inzwischen Tradition. Diese Tagungskooperation startete 2009 mit einem Versuch in Berlin, etablierte sich 2012 in Wien, 2014 folgte Winterthur, 2016 Bonn und 2018 traf man sich erneut in Wien. Zürich stand 2020 auf dem Programm - die Pandemie zerstörte alle Pläne. Vom 16.-18. Juni 2022 hofft die Schweizerische Medizinische Gesellschaft für Phytotherapie (SMGP) nun, diese internationale Tagung im zweiten Anlauf unter Dach und Fach zu bringen, um für 2024 den Weg für die Premiere in den Niederlanden frei zu machen. Heute, im Januar 2022, steht noch nicht fest, in welcher Form die Tagung stattfinden wird. Die 35. Schweizerische Jahrestagung für Phytotherapie konnte am 21. November 2021 mit über 200 Teilnehmenden mit Zertifikats- und Maskenpflicht ohne Zwischenfall durchgeführt werden (die ZPT berichtet noch). Dies stimmt zuversichtlich, dass das auch im Juni 2022 unter Einhaltung der dannzumal gültigen Vorgaben möglich sein wird. Eine weitere Voraussetzung ist, dass das Reisen innerhalb von Europa ohne Restriktionen erlaubt bleibt. Notfalls wird die Tagung als Videokonferenz präsentiert, eine erneute Verschiebung ist nicht geplant.

Das Programm von 2020 konnte für 2022 weitgehend übernommen werden. Die Themen sind auch zwei Jahre später hochaktuell. In der aufstrebenden Disziplin der Palliativ- und Supportivmedizin kann mit verfügbaren Zubereitungen therapiert werden. Dies aufzuzeigen, ist die Aufgabe der Referierenden, die aus Wissenschaft und Praxis rekrutiert wurden. Die SMGP freut sich besonders auf die Referate junger Phytotherapeutinnen, die mithelfen, den Generationenwechsel in der Phytotherapie voran zu bringen. Der zweite Kongresstag hat zum Ziel, das Spektrum der Phytotherapie zu erweitern. Es gibt noch einige Pflanzen, die z. B. vom HMPC monografiert sind, die aber nur wenig genutzt werden, so z. B. die Blutwurz. Auch das Potenzial der Harze erscheint nicht ausgeschöpft. Das Tagungsprogramm wird am Samstag abgerundet mit drei verschiedenen pharmakobotanischen Exkursionen. Die Betrachtung der Pflanzen an ihrem natürlichen Standort soll als Inspiration für vertiefte phytotherapeutische Diskussionen dienen.

Als Mitorganisator der Tagung wünsche ich mir, dass möglichst viele Mitglieder, Mitarbeitende und Vorstandsmitglieder der beteiligten Gesellschaften, aber auch von anderen Verbänden und Institutionen an dieser Tagung im Juni in Zürich mit dabei sind. Nur so kann der mit viel Aufwand organisierte Anlass eine Breitenwirkung entfalten. Zudem besteht - gerade nach zwei Jahren coronabedingter Einschränkungen - ein großer Wunsch, dass sich alle, die sich mit Phytotherapie beschäftigen, wieder regelmäßig treffen können, einerseits um sich besser kennen zu lernen, andererseits um sich gegenseitig auszutauschen. Dies scheint mir in diesen Tagen besonders wichtig.

Ich stehe am Ende meiner Laufbahn und gehe davon aus, dass die tetranationale Tagung unter dem Titel „Phytotherapie 2022 - innovativ“ die letzte solche Veranstaltung ist, bei der ich im Tagungspräsidium aktiv mit dabei bin. Deshalb würde es mich ganz besonders freuen, möglichst viele Wegbegleiter in Zürich treffen zu können. Deutlich mehr als 40 Jahre sind vergangen, seit ich meine ersten Kurzvorträge (Poster gab es damals noch keine!) an den Jahrestagungen der Gesellschaft für Arzneipflanzenforschung (wie sie damals hieß) in München und Zürich gehalten habe. Die Einfüh- 
rung der HPLC war damals ein großes Thema. Die Veränderungen, die seither stattgefunden haben, sind gewaltig. Die Fragen sind jedoch die gleichen geblieben: Qualität und Wirksamkeit der Arzneipflanzen sind auch mit Hightech-Methoden nicht abschließend festlegbar. Neue Erkenntnisse bringen mitunter große Fortschritte, revidieren aber auch oft, was festzustehen schien. Der klinische Wirksamkeitsnachweis in der Phytotherapie hat in den Jahrzehnten, auf die ich zurückblicke, große Fortschritte gemacht. Ich bin dankbar, dass ich in verschiedenen Bereichen mithelfen konnte, neue Erkenntnisse zu gewinnen und mit Tagungen, Workshops und Kongressen sowie im Rahmen des sehr erfolgreichen Schweizer Fähigkeitsprogramm Phytotherapie (SMGP) an der Vertiefung und Verbreitung neuer sowie bewährter, aber durch neue Untersuchungen gefestigter Erkenntnisse beitragen konnte. Deshalb: Willkommen in Zürich!

Die SMGP ist stolz, diesen Anlass an der Universität in Zürich durchführen zu dürfen. Wir unterstreichen damit die Verbindung der Phytotherapie zur Wissenschaft und möchten aufzeigen, dass der Phytotherapie ein angemessener Platz an den Hochschulen gebührt. Wir wissen uns dabei unterstützt vom Institut für komplementäre und integrative Medizin des Universitätsspitals unter der Leitung von Frau Professor Dr. med. Claudia Witt.

\section{Was erwarten die beteiligten Gesellschaften vom tetranationalen Kongress in Zürich?}

Am tetranationalen Phytotherapiekongress sind mittlerweile fünf Verbände beteiligt. Sie haben ihre Erwartungen an den Kongress in Zürich formuliert:

\section{ESCOP - European Scientific Cooperative on Phytotherapy}

Für die 2022 geplante Arbeit der ESCOP an weiteren Monografien sind die Neuigkeiten in den Vorträgen zu Ginkgo, Ingwer und Vogelknöterich im Rahmen der tetranationalen Tagung „Phytotherapie - 2022“ von ganz besonderem Interesse.

\section{GPT - Gesellschaft für Phytotherapie e.V}

Das Programm der tetranationalen Tagung in Zürich unterstreicht aus unserer Sicht die große Bedeutung seit Langem in der Ethnomedizin verwendeter, traditioneller Arzneipflanzen in der modernen wissenschaftlichen Phytotherapie. Gerade der Austausch über Ansätze zu einer möglichen Weiterentwicklung des Begriffes der Tradition, wie er in der Podiumsdiskussion erfolgen soll, sind für uns, neben allen anderen neuen Erkenntnissen zur Anwendung pflanzlicher Zubereitungen bei Mensch und Tier, von besonderer Bedeutung.

\section{NVF - Niederländische Gesellschaft für Phytotherapie}

Die NVF freut sich auf einen erfolgreichen und inspirierenden Kongress im Juni 2022 in Zürich. Wir interessieren uns vor allem für die Themen, die die Bedeutung der Phytotherapie in der Supportivmedizin bei onkologischen Patienten und in der Palliativmedizin erläutern. Durk Meijer präsentiert dabei eine Analyse zur Frage, wie Ingwer als Mittel gegen Erbrechen in der Chemotherapie eingesetzt werden kann.

\section{ÖGPHYT - Österreichische Gesellschaft für Phytotherapie}

Für den kommenden tetranationalen Kongress freut sich die ÖGPHYT über möglichst viele Teilnehmende und Posterbeiträge speziell aus Österreich. Besonders interessiert ist die ÖGPHYT an der Podiumsdiskussion und den Referaten zum Thema „Traditional Use“. Johannes Saukel präsentiert mit dem Fichtenharz ein traditionelles, österreichisches, pflanzliches Arzneimittel mit nachgewiesenem Potenzial in der Wundheilung. 


\section{SMGP - Schweizerische Medizinische Gesellschaft für Phytotherapie}

Phytotherapie in der Palliativ- und Supportivmedizin weckt zunehmend das Interesse auch unter jungen MedizinerInnen, die sich in diesem Bereich engagieren. Pflanzliche Arzneimittel finden ihren Platz und helfen, dank ihrer Multifunktionalität die manchmal riesigen Listen synthetischer Arzneimittel auf den Rezepten vieler PatientInnen zu entschlacken. In der Literatur ist bisher darüber noch nicht viel zu finden - mit umso mehr Spannung werden die Referate erwartet. Dass die Onkologie mit Referaten aus drei Ländern speziell beleuchtet wird, ist bemerkenswert.

\section{SMGPvet - Schweizerische Medizinische Gesellschaft für Phytotherapie, Sektion Veterinärmedizin}

In der SMGP hat sich im letzten Jahrzehnt eine aktive und lebendige Sektion für Veterinärmedizin mit rund 100 Mitgliedern etabliert. Sie versucht, national und international Inputs zu setzen, um die Phytotherapie in der Veterinärmedizin wieder zu verankern. Mit den Schwerpunkten am Freitag verbindet sich die Hoffnung, dass auch die anderen beteiligten Gesellschaften in diesem Umfeld aktiv werden. Dies ist besonders wichtig, weil in der neuen Europäischen Tierarzneimittelverordnung nahezu nichts zu pflanzlichen Tierarzneimitteln zu lesen ist. Dies könnte bis 2027 geändert werden, wenn die nötigen Daten aufbereitet sind. So steht die Bilanz zu einem mittlerweile zehnjährigen Forschungsprojekt in der Schweiz zur Erfassung der Anwendungstradition pflanzlicher Rezepturen in verschiedenen Regionen der Schweiz und benachbarter Länder im Mittelpunkt.

\section{Dank}

Der Dank geht an alle, die sich für die Phytotherapie engagieren. Für „Phytotherapie 2022“ gilt dies besonders für die Mitglieder des Tagungspräsidiums, Beatrix Falch und Michael Walkenhorst, aber auch an das Organisationskomitee sowie Marianne Ernst und Rachel Urenda von der Zürcher Hochschule für angewandte Wissenschaften in Wädenswil. Insbesondere Beatrix Falch leistet einen immensen Einsatz, ohne den diese Tagung nicht möglich wäre.

Beat Meier

Zur Tagungs-Website: phytotherapie2022.smgp.ch - oder via QR-Code (s. Banner unten rechts):

TETRANATIONALE TAGUNG PhYTOTHERAPIE 2022 36. Schweizerische Jahrestagung für Phytotherapie
Phytotherapie 2022 - innovativ 16. f18. Juni 2022, Universität Zürich hitp://phytotherapie2022.smgp.ch 